\title{
CURRENT GAIN IN UNIPOLAR PULSED DISCHARGE IN LOW-PRESSURE CARBON DIOXIDE
}

\author{
V.A. Lisovskiy, S.V. Dudin, N.N. Vusyk, V.A. Volkov, V.D. Yegorenkov, \\ A.N. Dakhov, P.A. Ogloblina \\ V.N. Karazin Kharkiv National University \\ 4 Svobody square, Karkiv 61022 \\ e-mail:lisovskiy@yahoo.com \\ Received 7 September, 2017; accepted 15 November, 2017
}

An unipolar low-pressure pulsed discharge (0.1-0.5 Torr) in $\mathrm{CO}_{2}$ has been investigated in the frequency range 20-200 kHz with duty cycle of 0.1 to 0.85 and voltage amplitude of $600-1000 \mathrm{~V}$. It was found that the current gain phenomenon, observed earlier in the mid-frequency pulsed discharges in other gases, also occurs for a discharge in $\mathrm{CO}_{2}$ with the gain of 1-2. The gain factor decreases with the discharge current growth, both due to increase of the voltage amplitude and due to increase of carbon dioxide pressure. It is shown that the shape of the oscillogram of the pulsed discharge current is determined primarily by the duration of the plasma phase and is almost independent on the duration of the afterglow phase. A physical mechanism of the phenomenon of current gain in a pulsed discharge is discussed. It is concluded that the most important factor in this mechanism is the heating of the gas in the discharge gap during the plasma phase of the discharge, which leads to depletion of neutral gas molecule concentration and, as a consequence, to ionization rate decrease.

KEYWORDS: gas discharge, plasma, carbon dioxide, pulsed discharge, current gain

\section{ПІДСИЛЕННЯ СТРУМУ В УНІПОЛЯРНОМУ ІМПУЛЬСНОМУ РОЗРЯДІ У ВУГЛЕКИСЛОМУ ГАЗІ НИЗЬКОГО ТИСКУ}

В.О. Лісовський, С.В. Дудін, М.М. Вусик, В.А. Волков, В.Д. Сгоренков, О.М. Дахов, П.О. Оглобліна

Харківский національний університет імені В.Н. Каразіна майдан Свободи 4, Харків, 61022, Украӥна

Досліджено уніполярний імпульсний розряд низького тиску $\left(0,1-0,5\right.$ Торр) в $\mathrm{CO}_{2}$ в діапазоні частот 20-200 кГц при коефіцієнті заповнення імпульсів від 0,1 до 0,85 і амплітуді напруги 600-1000 В. Виявлено, що ефект підсилення струму, що спостерігався раніше в середньочастотних імпульсних розрядах в інших газах, має місце $\mathrm{i}$ для розряду в $\mathrm{CO}_{2} 3$ коефіцієнтом підсилення 1-2. При цьому коефіцієнт підсилення зменшується з ростом струму розряду, як при збільшенні амплітуди імпульсної напруги, так і при збільшенні тиску вуглекислого газу. Показано, що форма осцилограми струму імпульсного розряду визначається в першу чергу тривалістю плазмової фази і майже не залежить від тривалості фази післясвітіння. Обговорюється фізичний механізм явища підсилення струму в імпульсному розряді. Зроблено висновок про те, що найважливішим фактором у цьому механізмі $є$ нагрів газу в розрядному проміжку протягом плазмової фази розряду, який призводить до локального падіння концентрації нейтральних молекул газу і, як наслідок, до зниження швидкості іонізації.

КЛЮЧОВІ СЛОВА: газовий розряд, плазма, вуглекислий газ, імпульсний розряд, підсилення струму

\section{УСИЛЕНИЕ ТОКА В УНИПОЛЯРНОМ ИМПУЛЬСНОМ РАЗРЯДЕ В УГЛЕКИСЛОМ ГАЗЕ НИЗКОГО ДАВЛЕНИЯ}

В.А. Лисовский, С.В. Дудин, Н.Н. Вусык, В.А. Волков, В.Д. Егоренков, А.Н. Дахов, П.А. Оглоблина

Харьковский национальный университет имени В.Н. Каразина пл. Свободыл 4, Харьков, 61022, Украина

Исследован униполярный импульсный разряд низкого давления $\left(0,1-0,5\right.$ Торр) в $\mathrm{CO}_{2}$ в диапазоне частот 20-200 кГц при коэффициенте заполнения импульсов от 0,1 до 0,85 и амплитуде напряжения 600-1000 В. Обнаружено, что эффект усиления тока, наблюдавшийся ранее в среднечастотных импульсных разрядах в других газах, имеет место и для разряда в $\mathrm{CO}_{2}$ с коэффициентом усиления 1-2. При этом коэффициент усиления уменьшается с ростом тока разряда, как при увеличении амплитуды импульсного напряжения, так и при повышении давления углекислого газа. Показано, что форма осциллограммы тока импульсного разряда определяется в первую очередь длительностью плазменной фазы и почти не зависит от длительности фазы послесвечения. Обсуждается физический механизм явления усиления тока в импульсном разряде. Сделан вывод о том, что важнейшим фактором в этом механизме является нагрев газа в разрядном промежутке в течение плазменной фазы разряда, который приводит к локальному падению концентрации нейтральных молекул газа и, как следствие, к снижению скорости ионизации.

КЛЮЧЕВЫЕ СЛОВА: газовый разряд, плазма, углекислый газ, импульсный разряд, усиление тока

Газоразрядная плазма широко применяется во множестве технологических процессов - для травления полупроводниковых материалов и осаждения пленок при производстве изделий микроэлектроники, солнечных панелей, упрочнения металлических изделий и инструментов, накачки газоразрядных лазеров, плазменной стерилизации и так далее. Часто в таких технологиях используют разряды постоянного тока, а также высокоча- 
стотные разряды, которые имеют множество недостатков. В разряде постоянного тока могут наблюдаться сильный нагрев обрабатываемых образцов и дугообразование, что может вызвать их термическое разрушение. Высокочастотные разряды нуждаются в дорогостоящих и сложных в эксплуатации генераторах и системах согласования. Поэтому в последнее время большое внимание стало уделяться импульсным среднечастотным (десятки-сотни кГц) разрядам. В отличие от высокочастотного и тлеющего разрядов, среднечастотные импульсные разряды изучены значительно меньше.

Как показывают результаты работ [1-9], импульсные разряды обладают рядом специфических особенностей, которые могут быть полезны в разнообразных приложениях. Например, в работе [3] микросекундный импульсный тлеющий разряд применялся к источнику плазмы типа Грима, который используется для ионизации анализируемых веществ в масс-спектрометрии. Продемонстрировано существенное улучшение аналитических свойств масс-спектрометра с этим разрядом по сравнению со стационарным разрядом постоянного тока.

Следует отметить, что в работах [2-9] было обнаружено, что в импульсном разряде в азоте, аргоне, кислороде и $\mathrm{CF}_{4}$ средний ток в течение плазменной фазы может быть заметно выше, чем ток тлеющего разряда при том же напряжении на электродах. Это интересное свойство импульсного разряда было названо эффектом усиления тока, который состоит в том, что импульсное питание разряда (иными словами, периодическое прерывание напряжения, приложенного к электродам тлеющего разряда), приводит не к уменьшению разрядного тока, а к его многократному росту в сравнении с разрядом постоянного тока с таким же приложенным напряжением. В работе [10] были исследованы разряды в различных молекулярных газах, $\mathrm{O}_{2}, \mathrm{~N}_{2}, \mathrm{CF}_{4}, \mathrm{SF}_{6}$, и были обнаружены коэффициенты усиления тока от 1-2 для кислорода и азота до 9 для элегаза.

В настоящее время большое внимание уделяется процессам, происходящим в плазме в $\mathrm{CO}_{2}$, в связи с тем, что этот газ вызывает парниковый эффект в земной атмосфере, и разработка эффективных технологий конверсии углекислого газа является одной из приоритетных задач физики газоразрядной плазмы [11-17]. Кроме того, $\mathrm{CO}_{2}$ широко распространен в атмосферах планет и спутников Солнечной системы, и в будущих космических миссиях к этим планетам планируется использование конверсии углекислого газа для получения СО в качестве топлива и кислорода для дыхания людей [18].

В одной из последних статей [18] было показано, что использование импульсного разряда является одним из наиболее перспективных направлений для технологий конверсии $\mathrm{CO}_{2}$. Однако импульсные разряды в углекислом газе еще недостаточно изучены, в частности, отсутствуют работы по изучению эффекта усиления тока. Поэтому целью нашей работы было исследование явления усиления тока в плазменной фазе униполярного импульсного разряда низкого давления в $\mathrm{CO}_{2}$.

\section{ОПИСАНИЕ ЭКСПЕРИМЕНТАЛЬНОЙ УСТАНОВКИ}

Блок-схема устройства, на котором проводились эксперименты, показана на рис. 1. Установка была аналогична описанной в [10]. Разрядная трубка цилиндрической формы имела внутренний диаметр 56 мм. Внутри трубки располагались плоские анод и катод из нержавеющей стали, все эксперименты проводились при расстоянии между электродами 20 мм. К катоду прикладывалось отрицательное импульсное униполярное напряжение в диапазоне $600-1000$ В, анод был заземлен. Частота импульсов изменялась в диапазоне 20-200 кГц при коэффициенте заполнения $D$ от 0,1 до 0,85 . Для измерения напряжения между электродами и разрядного тока использовался осциллограф PCS500 (Velleman Instruments), сигналы с него подавались на персональный компьютер. Напряжение разряда измерялось с помощью высоковольтного делителя с частотной компенсацией, а токоизмерительный резистор 10 Ом подключался последовательно к разрядному контуру со стороны анода. Диапазон измеренных значений тока разряда не превышал 100 мА. Эксперименты проводились в углекислом газе в диапазоне давлений $0,1-0,5$ Торр. Для определения давления газа использовался емкостный датчик давления типа MKS Baratron 627 с максимальным измеряемым давлением 10 Торр.

\section{ЭКСПЕРИМЕНТАЛЬНЫЕ РЕЗУЛЬТАТЫ}

При описании результатов экспериментов будем использовать терминологию, принятую в работах [8-10]. В осциллограммах тока были выделены две фазы: плазменная фаза и фаза послесвечения. Наблюдались следующие этапы плазменной фазы: 1. Импульс тока смещения длительностью примерно 0,5-1 мкс; 2. Этап роста тока, длительность которого зависела от сорта газа, длительности плазменной фазы и давления; 3. Плато (заметно выраженное лишь для $\mathrm{CF}_{4}$ и отсутствующее в азоте и кислороде); 4. Уменьшение тока, длившееся десятки микросекунд, до уровня, соответствующего разряду с постоянным напряжением.

Ключевым параметром, который исследуется в настоящей работе, является усиление тока в импульсном разряде. Для того, чтобы дать ему количественное определение, рассмотрим характерные осциллограммы напряжения и тока, показанные на рис.2. Из рисунка видно, что напряжение на катоде увеличивается от нуля до максимальной величины (в данном случае до -600 В) примерно в течение 1 мкс, при этом скорость его роста со временем уменьшается. На осциллограмме тока в начале периода виден пик, вызванный током смещения, который пропорционален скорости роста напряжения. После этого ток уменьшается, оставаясь заметно выше тока стационарного разряда $I_{d c}$ при данном напряжении. На рис.2 горизонтальной пунктирной линией показана величина импульсного тока $I_{p}$ в конце плазменной фазы. Именно отношение $I_{p} / I_{d c}$ мы будем считать численным 
значением коэффициента усиления тока в импульсном разряде.

Отметим, что результаты работы относятся к плазменной фазе импульсного разряда при параметрах, когда между электродами формируется разряд с хорошо выраженными катодным слоем и отрицательным свечением, а при более длинных зазорах между электродами и высоких давлениях газа появляются также темное фарадеево пространство, положительный столб и анодный слой. Плазменная фаза заканчивается в результате уменьшения напряжения на катоде до нуля, и разряд переходит в фазу послесвечения или распадающейся плазмы. Однако, как свидетельствуют результаты работы [10], в микросекундных импульсных разрядах в течение послесвечения плазма не успевает заметно распасться, что значительно облегчает формирование разряда при подаче на катод очередного импульса напряжения.

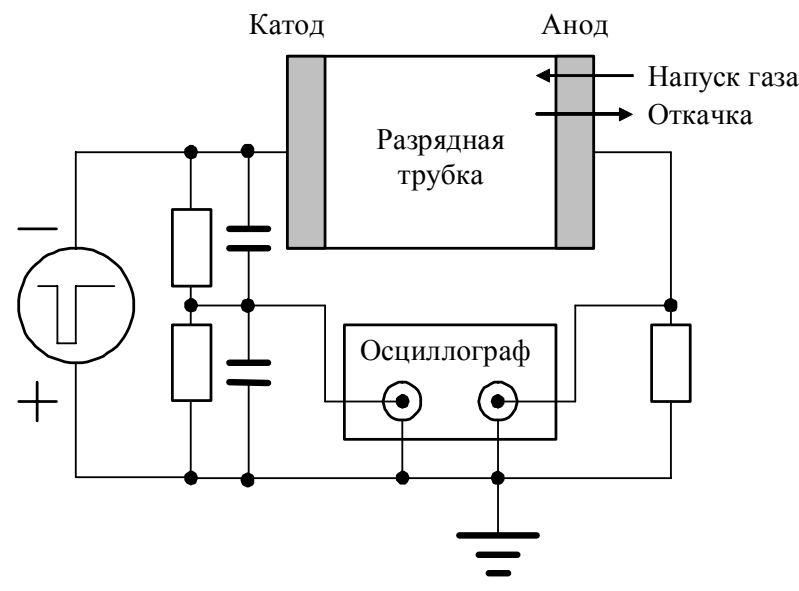

Рис. 1. Блок-схема экспериментальной установки

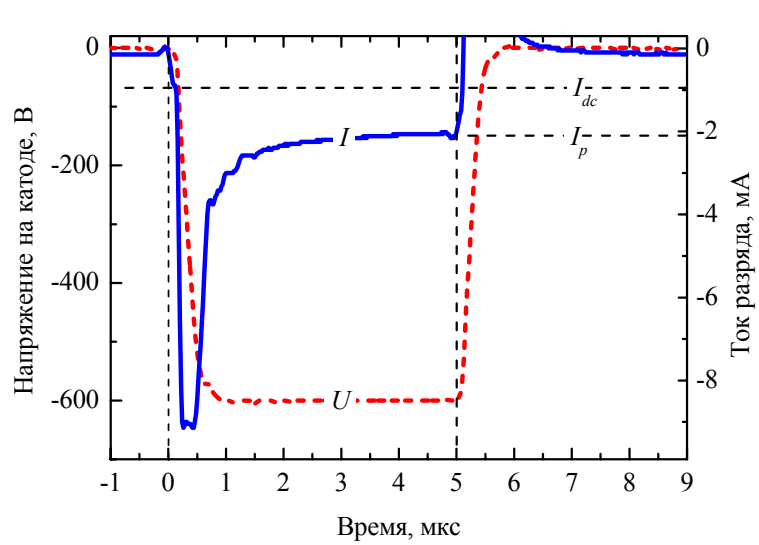

Рис.2. Осциллограммы напряжения и тока униполярного импульсного разряда при давлении 0,1 Торр, амплитуде приложенного напряжения 600 В, частоте 100 кГц, и коэффициенте заполнения 0,5

Рассмотрим влияние коэффициента заполнения импульсов $D$ на ток импульсного разряда. Соответствующие осциллограммы тока приведены на рис.3.
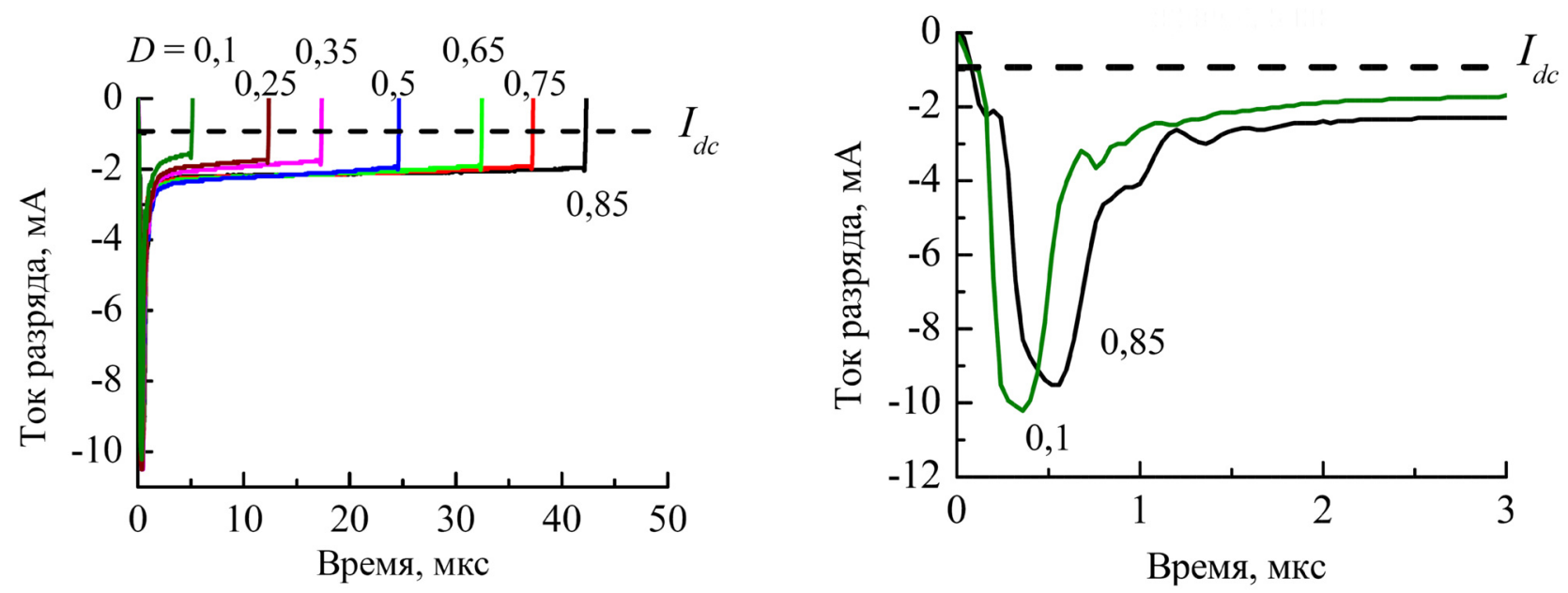

Рис.3. Осциллограммы тока униполярного импульсного разряда при давлении 0,1 Торр, амплитуде приложенного напряжения $600 \mathrm{~B}$, частоте 20 кГц и различных коэффициентах заполнения. Пунктирная линия - величина постоянного тока тлеющего разряда для напряжения $600 \mathrm{~B}$

На этом рисунке, а также на рис. 4, показано поведение тока как в течение всего импульса напряжения, так и в самом его начале. Из рисунка видно, что наибольшая амплитуда начального пика тока наблюдается при небольшом коэффициенте заполнения $D=0,1$, а ток для $D=0,85$ достигает максимума на 0,2 мкс позже, что связано с зависимостью от $D$ крутизны фронта импульса используемого нами генератора. В течение плазменной фазы ток для короткого импульса при $D=0,1$ быстро уменьшается (при длительных импульсах ток со временем уменьшается гораздо медленнее) и в конце импульса составляет $80 \%$ от тока для $D=0,85$. По-видимому, это объясняется тем, что при больших коэффициентах заполнения суммарный энерговклад в плазму больше, что обеспечивает более высокую ее концентрацию, и, следовательно, более высокий ток. Однако при изменении $D$ от 0,1 до 0,85 , то есть в 8,5 раз, ток меняется только на $20 \%$. Это свидетельствует о том, что при данных усло- 
виях разряд близок к равновесному, то есть процессы рождения заряженных частиц в плазменной фазе уравновешиваются их уходом в основном именно в плазменной фазе, а уход заряженных частиц из разряда в фазе послесвечения сильно замедляется в связи с резким падением электронной температуры после окончания плазменной фазы [10].
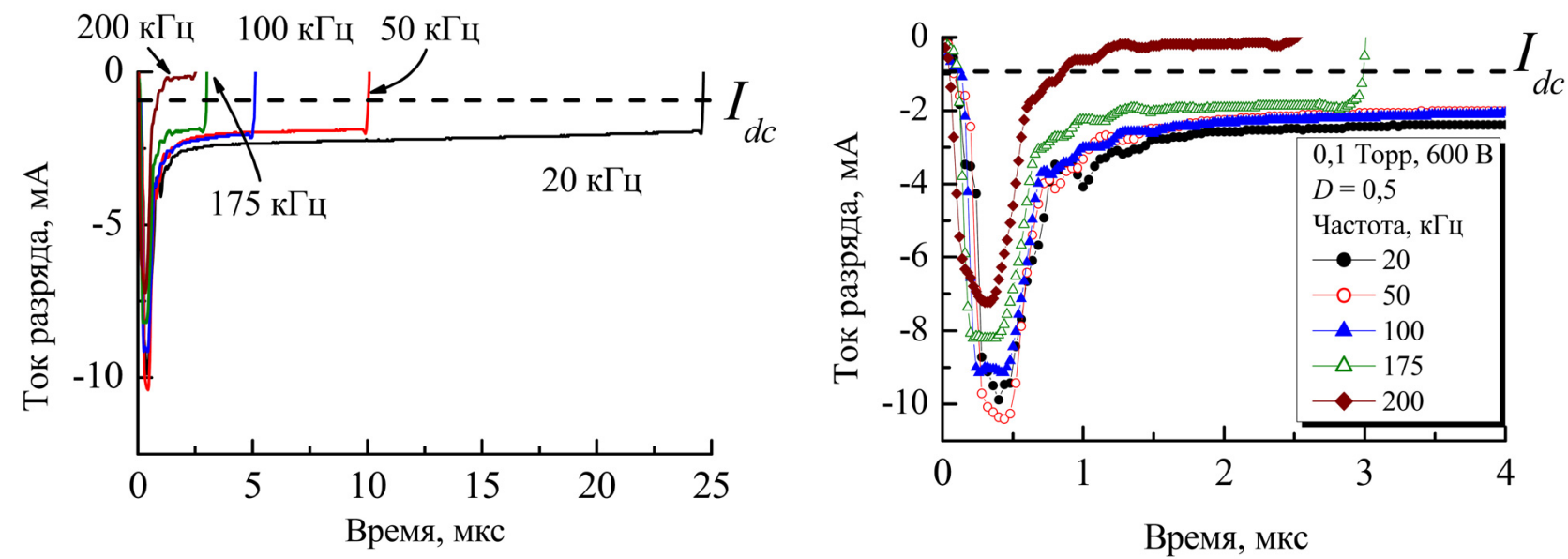

Рис.4. Осциллограммы тока униполярного импульсного разряда при давлении 0,1 Торр, амплитуде приложенного напряжения $600 \mathrm{~B}$, коэффициенте заполнения 0,5 и различных частотах. Пунктирная линия - величина постоянного тока тлеющего разряда для напряжения $600 \mathrm{~B}$.

На рис.4 показано влияние частоты на осциллограммы тока при фиксированных значениях давления газа, приложенного напряжения и коэффициента заполнения (представлены только токи для плазменной фазы одного импульса, чтобы не загромождать рисунок данными). Из рисунка, во-первых, видно, что повышение частоты приводит к уменьшению как величины начального пика тока, так и разрядного тока в течение всей плазменной фазы. Во-вторых, из рис.4 следует, что почти при всех частотах ток импульсного разряда превышает ток тлеющего разряда при постоянном напряжении на электродах. Исключением является только осциллограмма тока для частоты 200 кГц, которая во второй половине плазменной фазы проходит ниже (по абсолютной величине), чем ток тлеющего разряда $I_{d c}$. Это объясняется тем, что напряжение 600 В близко к кривой погасания импульсного разряда для этой частоты, соответственно, была низкой плотность плазмы. При более высокой частоте для указанных величин напряжения и давления газа импульсный разряд не горел.

Зависимость тока $I_{p}$ от импульсного напряжения на электродах показана на рис.5. На этом же рисунке представлены зависимость тока $I_{d c}$ тлеющего разряда от постоянного (не импульсного) напряжения, а также отношение импульсного и постоянного токов $I_{p} / I_{d c}$. Из рисунка видно, что и импульсный, и постоянный токи монотонно увеличиваются с ростом напряжения на электродах. Однако скорости их роста различны, поэтому отношение токов с повышением напряжения от 600 В до 1000 В снижается от 2 до 1. Это указывает на то, что чем выше приложенное напряжение, тем быстрее после начального всплеска импульсный ток стремится к току, который соответствует тлеющему разряду с постоянным напряжением.

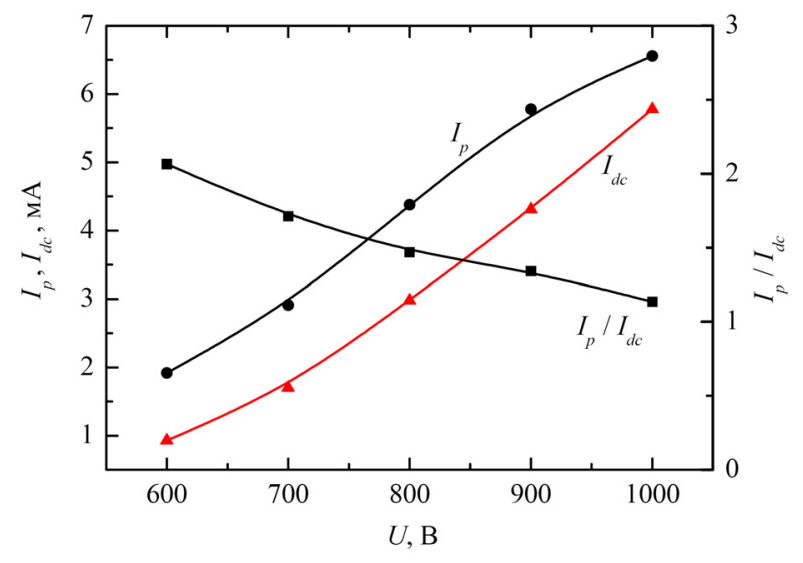

Рис. 5. Зависимость импульсного тока $I_{p}$ в конце плазменной фазы, тока тлеющего разряда $I_{d c}$, а также их отношения $I_{p} / I_{d c}$ от напряжения на электродах при давлении газа 0,5 Торр, частоте импульсного напряжения 20 кГц и коэффициенте заполнения 0,5 .

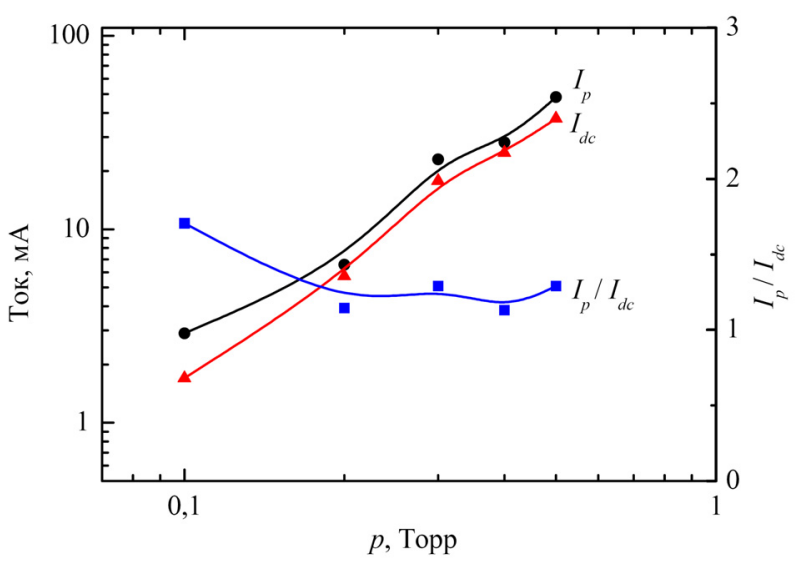

Рис. 6. Зависимость импульсного тока $I_{p}$ в конце плазменной фазы, тока тлеющего разряда $I_{d c}$, а также их отношения $I_{p} / I_{d c}$ от давления газа при напряжении на электродах $700 \mathrm{~B}$, частоте импульсного напряжения 20 кГц и коэффициенте заполнения 0,5 . 
Таким образом, мы можем сделать важный вывод: при более высоких импульсных напряжениях формирование стационарного разряда происходит быстрее. С ростом давления газа формирование разряда ускоряется, о чем свидетельствует приближение к единице отношения токов $I_{p} / I_{d c}$ при высоких давлениях (рис.6). Следует отметить, что с уменьшением давления уменьшается и величина разрядного тока, то есть, как и в случае зависимости от напряжения, наибольшее усиление тока импульсного разряда наблюдается при небольших токах.

\section{ОБСУЖДЕНИЕ РЕЗУЛЬТАТОВ}

Одним из наиболее важных выводов, которые были сделаны в предыдущем разделе на основе анализа экспериментальных результатов, является то, что в углекислом газе в рассмотренном диапазоне параметров ток униполярного импульсного разряда в плазменной фазе практически всегда превышает ток стационарного тлеющего разряда при том же напряжении. Отношение токов $I_{p} / I_{d c}$ в работах [8-10] рассматривается как “коэффициент усиления тока в импульсном разряде". Как уже было сказано во Введении, усиление тока в импульсном разряде экспериментально наблюдалось для различных газов, были исследованы основные закономерности этого явления, однако, его физический механизм до конца не ясен. Упомянутые выше исследования других авторов были выполнены в азоте, аргоне, $\mathrm{CF}_{4}$ и $\mathrm{SF}_{6}$. Анализ различных процессов в этих газах показал, что в эффект усиления тока могут вносить вклад импульсный нагрев газа в разрядном промежутке, диссоциация молекул, образование отрицательных ионов в электроотрицательных газах.

Проведем анализ возможных процессов, принимающих участие в униполярном импульсном разряде в углекислом газе. Для удобства отдельно рассмотрим плазменную фазу и послесвечение (фазу с распадающейся плазмой).

\section{Плазменная фаза}

1. Переходные процессы в начале плазменной фазы, когда формируются катодный слой и отрицательное свечение.

В предыдущей работе [10] мы с помощью численного моделирования показали, что после выключения напряжения электроны, а также положительные (и в случае электроотрицательных газов отрицательные) ионы частично заполняют катодный слой. В начале очередного импульса напряжения эти заряженные частицы должны в течение какого-то времени перераспределяться внутри слоя: электроны и отрицательные ионы - выталкиваться из слоя; положительные ионы - устремляться к катоду. Сталкиваясь с его поверхностью, ионы высвободят вторичные электроны, которые создадут новые электронные лавины в слое. Это должно привести к заметному росту разрядного тока. Однако, как было показано в [10], время формирования катодного слоя составляет небольшие доли микросекунды, и в течение времени нарастания напряжения на электродах катодный слой претерпевает только небольшие изменения его толщины и плотности плазмы на его границе. Поэтому данный механизм не может объяснить заметное усиление тока в импульсном разряде.

2. Диссоциация молекул углекислого газа.

Образующиеся при диссоциации молекул углекислого газа атомы кислорода О и молекулы СО имеют потенциалы ионизации, близкие к $\mathrm{CO}_{2}$. Так, для $\mathrm{CO}_{2}$ потенциал ионизации равен 13,77 эВ [19], для $\mathrm{CO}-14,01$ эВ, для атомарного кислорода - 13,62 эВ. Поэтому образующиеся при диссоциации молекулы СО не являются легко ионизуемой добавкой к основному газу $\mathrm{CO}_{2}$, а различие в 0,15 эВ между потенциалами ионизации $\mathrm{CO}_{2}$ и атомов О слишком мало, чтобы существенно повлиять на характеристики формирующегося разряда. Однако потенциал ионизации молекул $\mathrm{O}_{2}$ равен 12,07 эВ, поэтому наличие достаточно высокой их концентрации может облегчить зажигание и поддержание плазменной фазы. В работе [20] авторы с помощью глобальной модели оценили состав газовой среды и концентрации заряженных частиц в диапазоне давлений 0,001 - 0,6 Торр. Для давления 0,1 Торр они получили, что в горящем тлеющем (не импульсном) разряде концентрации нейтральных молекул $\mathrm{CO}_{2}$ и $\mathrm{CO}$ примерно равны $10^{15} \mathrm{~cm}^{-3}$, концентрации молекул $\mathrm{O}_{2} 4 \cdot 10^{14} \mathrm{~cm}^{-3}$ и атомов $\mathrm{O} 1,5 \cdot 10^{14} \mathrm{~cm}^{-3}$. Следовательно, молекулы $\mathrm{O}_{2}$, сохранившиеся в зазоре между электродами в течение послесвечения, могут давать существенный вклад в формирование и горение разряда.

3. Диссоциативное прилипание электронов с последующим их отлипанием.

Диссоциативное прилипание $\left(\mathrm{CO}_{2}+\mathrm{e}^{-} \rightarrow \mathrm{CO}+\mathrm{O}^{-}\right)$начинается с пороговой энергии электронов 3,85 эВ $[21,22]$. Это означает, что отрицательные ионы могут появляться только в течение плазменной фазы. Энергия связи электрона с атомом кислорода (энергия сродства) равна 1,47 эВ [23]. Поэтому для отлипания (отрыва) связанного электрона от отрицательного иона нужно, чтобы либо электроны в начале формирования плазменной фазы имели достаточно высокую для этого энергию (что выполняется в плазменной фазе), либо отрицательный ион $\mathrm{O}^{-}$должен столкнуться с атомарным кислородом либо молекулой СО (что может происходить как в плазменной фазе, так и в послесвечении). В результате таких реакций при слиянии двух атомов кислорода в молекулу $\mathrm{O}_{2}\left(\mathrm{O}^{-}+\mathrm{O} \rightarrow \mathrm{O}_{2}+\mathrm{e}^{-}\right)$или $\mathrm{O}$ и $\mathrm{CO}$ в молекулу $\mathrm{CO}_{2}\left(\mathrm{O}^{-}+\mathrm{CO} \rightarrow \mathrm{CO}_{2}+\mathrm{e}^{-}\right)$выделяется энергия связи 3,6 эВ и 4 эВ [23], соответственно, которой достаточно для отрыва электрона. При условиях, описанных в [20], концентрация отрицательных ионов $\mathrm{O}^{-}$равна $4 \cdot 10^{10} \mathrm{~cm}^{-3}$, а плотность электронов $5 \cdot 10^{10} \mathrm{~cm}^{-3}$. Скорость рождения электронов в $1 \mathrm{~cm}^{3}$ из-за отлипания (равная скорости потерь отрицательных ионов с отрицательным знаком) при столкновениях $\mathrm{O}^{-}+\mathrm{O}$ определяется формулой 


$$
\left(\frac{d n_{e}}{d t}\right)_{d}=-\left(\frac{d n_{-}}{d t}\right)_{d}=v_{d} \cdot n_{-}=K_{d} \cdot N_{O} \cdot n_{-},
$$

где $K_{d}=2 \cdot 10^{-10} \mathrm{~cm}^{3} / \mathrm{c}$ - константа скорости и $v_{d}$ - частота данной реакции [23], $N_{O}$ - концентрация атомов кислорода, $n_{-}$- плотность отрицательных ионов. Тогда частота отлипания равна $v_{d}=2 \cdot 10^{-10} \cdot 1,5 \cdot 10^{14}=3 \cdot 10^{4} \mathrm{c}^{-1}, \mathrm{a}$ время отлипания $\tau_{d}=3,3 \cdot 10^{-5}$ c. Аналогично для концентрации CO $10^{15} \mathrm{~cm}^{-3}$ [20] и константы $K_{d}=4 \cdot 10^{-10} \mathrm{~cm}^{3} / \mathrm{c}$ [24] скорости реакции $\mathrm{O}^{-}+\mathrm{CO} \rightarrow \mathrm{CO}_{2}+\mathrm{e}^{-}$легко получить время отлипания $\tau_{d}=2,5 \cdot 10^{-6}$ с. Поэтому отлипание при столкновениях $\mathrm{O}^{-}+\mathrm{CO}$ может давать существенный вклад в поддержание разряда в течение плазменной фазы (которая при частоте 20 кГц длится 50 микросекунд). Однако процесс столкновений $\mathrm{O}^{-}+\mathrm{O}$ слишком редко заканчивается отрывом связанного электрона от отрицательного иона, чтобы играть в импульсном разряде значительную роль.

4. Нагрев нейтрального газа.

Большой цикл работ (см., например, [2-6]) посвящен формированию униполярного импульсного разряда в аргоне и азоте. При этом в качестве одного из ключевых факторов, оказывающих влияние на формирование параметров импульсного разряда, рассматривается изменение температуры нейтрального газа в разрядном промежутке под воздействием энергии, вкладываемой в плазму импульсного разряда. Так, в докладе [2] показано, что изменение напряжения, давления и коэффициента заполнения оказывает основное влияние на температуру газа. Изменение тока с коэффициентом заполнения можно объяснить влиянием разрядной мощности на температуру газа. Когда коэффициент заполнения увеличивается, среднее поглощение мощности также возрастает, что приводит к нагреву плазмы. При постоянном давлении, в соответствии с термодинамическим законом $p=n k T$, увеличение температуры газа приводит к уменьшению концентрации атомов и, следовательно, понижению разрядного тока.

Авторы работы [4] обнаружили и исследовали волны давления газа как в униполярном импульсном разряде в источнике Грима с помощью встроенного микрофона. Обнаружено, что первая и вторая гармоники колебаний давления хорошо согласуются с резонансной частотой (2,7 кГц) разрядной камеры. Формирование волны давления газа происходит на краях разряда. Авторы [4] предположили, что механизмом генерации волн давления является нагрев газа в плазме с его последующим расширением и появлением звуковых волн в разрядной камере на ее естественной резонансной частоте. На заднем фронте импульса волны генерируются при охлаждении и сжатии газа.

Выше мы уже сказали, что катодный слой после подачи на электроды напряжения быстро (за доли микросекунды) восстанавливается после некоторого его распада в течение послесвечения. В нем развиваются электронные лавины, а к катоду из всего объема катодного слоя и части отрицательного свечения идет поток положительных ионов. Эти ионы движутся в сильном электрическом поле, набирают энергию, которой эффективно обмениваются с нейтральными молекулами при столкновениях с ними (как упругие шары, а также при перезарядке). Происходит нагрев нейтрального газа вблизи поверхности катода. Так как толщина катодного слоя (примерно 5 мм) мала по сравнению с расстоянием между электродами, то для поддержания постоянного (по всей разрядной камере) давления часть молекул газа удаляется со скоростью звука. Элементарные оценки дают, что молекулы газа пересекают весь катодный слой за время порядка нескольких микросекунд, что сравнимо со временем снижения разрядного тока в течение плазменной фазы. При катодном падении напряжения 500 В, приложенном к катодному слою толщиной $5 \mathrm{mM}$, и плотности разрядного тока $1 \mathrm{~mA} / \mathrm{cm}^{2}$, примерно половина поглощаемой в слое энергии идет на нагрев газа в нем, что позволяет оценить плотность мощности порядка $0,5 \mathrm{BT} / \mathrm{cm}^{3}$. Тогда в течение 25 мкс в катодном слое в плазменной фазе газом поглощается энергия $12,5 \mu Д ж / \mathrm{cm}^{3}$, что составляет примерно 25\% полной кинетической энергии молекул, находящихся в катодном слое при температуре газа $300 \mathrm{~K}$. Поэтому этот эффект нагрева газа должен играть существенную роль в плазменной фазе импульсного разряда.

Отметим, что даже небольшой нагрев газа в катодном слое с последующим уходом части молекул из него может значительно повлиять на полный разрядный ток. В катодном слое присутствует сильное электрическое поле, которое сообщает электронам большую энергию. Длина свободного пробега электронов в $\mathrm{CO}_{2}$ при давлении 1 Торр оценивается равной $0,03 \mathrm{~cm}=0,3$ мм [23], а при 0,1 Торр будет 3 мм. Это означает, что электроны, пересекающие катодный слой, на его протяжении испытывают только несколько столкновений. Соответственно, чем меньше молекул газа электрон сможет ионизовать в катодном слое, тем меньше электронов выйдет из слоя в отрицательное свечение и потеряет там энергию на ионизацию. Поэтому даже небольшое уменьшение концентрации молекул газа из-за их нагрева в катодном слое может заметно уменьшить величину разрядного тока.

На самом деле, в горящем тлеющем разряде (с постоянным напряжением на электродах) газ успевает прогреться не только в катодном слое, но и во всей разрядной камере. В импульсном разряде в течение послесвечения газ успевает немного остыть, поэтому после подачи напряжения на электроды наблюдается максимально возможный (для данного напряжения) ток. И из-за описанного выше нагрева газа в катодном слое ток постепенно уменьшается, стремясь к величине тока, соответствующей тлеющему разряду. 


\section{Послесвечение}

После выключения напряжения на электродах наступает фаза с распадающейся плазмой - послесвечение. Если в течение этой фазы плазма успеет распасться вследствие различных причин - понадобится заново осуществлять пробой промежутка при подаче на электроды очередного импульса напряжения. Здесь мы проанализируем механизмы потерь заряженных частиц в послесвечении и оценим их характерное время. В послесвечении температура электронов быстро (за единицы микросекунд) уменьшается, но не до нуля, а сохраняется примерно равной 0,1 эВ в течение десятков микросекунд [24].

1. Распад плазмы из-за амбиполярной диффузии.

Как было сказано выше, $\mathrm{CO}_{2}$ - это слабо электроотрицательный газ, т.к. в нем присутствуют отрицательные ионы $\mathrm{O}^{-}$. Как в плазменной фазе, так и в послесвечении большую роль в процессе потерь заряженных частиц играет амбиполярная диффузия. В электроположительной плазме, состоящей только из электронов и положительных ионов, эти заряженные частицы движутся к стенкам камеры с одинаковыми скоростями и общим для них коэффициентом амбиполярной диффузии. Однако в плазме, содержащей еще и отрицательные ионы, коэффициенты амбиполярной диффузии различны для каждого сорта заряженных частиц. Так, для положительных ионов имеем формулу для коэффициента амбиполярной диффузии [25-27]

$$
D_{a+}=D_{+} \cdot \frac{1+\gamma+2 \alpha \gamma}{1+\alpha \lambda} \cdot \frac{1+\alpha \cdot \mu_{-} / \mu_{e}}{1+\alpha \cdot \mu_{-} / \mu_{e}+(1+\alpha) \cdot \mu_{+} / \mu_{e}},
$$

где $\gamma=T_{e} / T_{i}, T_{e}$ - температура электронов, $T_{i}$ - температура положительных и отрицательных ионов, которая обычно предполагается равной температуре нейтрального газа, $\alpha=n\lrcorner n_{e}$ - отношение концентраций отрицательных ионов и электронов, $D_{+}, D_{e}, \mu_{+}, \mu_{\mathrm{e}}$ - коэффициенты свободной диффузии $(D)$ и подвижность $(\mu)$ для ионов и электронов, соответственно. Приведем эти величины для давления $\mathrm{CO}_{2}$, равного 1 Торр. Для электронов подвижность $\mu_{e}=5,3 \cdot 10^{5} \mathrm{~cm}^{2}$ Торр/(В с) и коэффициент свободной диффузии $D_{e}=5,3 \cdot 10^{4} \mathrm{~cm}^{2}$ Торр/с были взяты из работы [28]. Доминирующим положительным ионом является $\mathrm{CO}_{2}^{+}$[20], подвижность его $\mu_{+}=844 \mathrm{~cm}^{2} \mathrm{Topp} /(\mathrm{B} \mathrm{c})$ в собственном газе была определена как среднее значение, полученное в работах $[29,30]$, коэффициент диффузии найден с помощью соотношения Эйнштейна $\left(D_{+} / \mu_{+}=T_{+}\right)$и для температуры ионов $T_{+}=300 \mathrm{~K}=0,026$ эВ он равен $D_{+}=21,9 \mathrm{~cm}^{2} \mathrm{Topp} / \mathrm{c}$. Для отрицательных ионов $\mathrm{O}^{-}$подвижность $\mu_{-}=2,43 \cdot 10^{3} \mathrm{~cm}^{2} \mathrm{Topp} /(\mathrm{B} \mathrm{c)}$ была взята из работ $[31,32]$.

Мы уже указали, что в работе [20] были найдены концентрации отрицательных ионов $\mathrm{O}^{-} 4 \cdot 10^{10} \mathrm{~cm}^{-3}$ и электронов $5 \cdot 10^{10} \mathrm{~cm}^{-3}$. В этом случае $\alpha=n / n_{e}=4 / 5=0,8$. Тогда с помощью формулы (2) определим коэффициент амбиполярной диффузии $D_{a^{+}}=58,9 \mathrm{~cm}^{2} / \mathrm{c}$. В случае двухкомпонентной электроположительной плазмы (состоящей только из электронов и ионов) коэффициент амбиполярной диффузии будет равен $D_{a}=106,3 \mathrm{~cm}^{2} / \mathrm{c}$, то есть, почти вдвое больше, чем в для плазмы с отрицательными ионами.

Теперь оценим время ухода заряженных частиц на стенки и электроды в послесвечении. Частота таких потерь равна

$$
v_{d}=\frac{D_{a+}}{\Lambda^{2}},
$$

где $\Lambda$ - диффузионная длина, которая для трубки радиусом $R$ при расстоянии между электродами $L$ описывается формулой

$$
\frac{1}{\Lambda^{2}}=\left(\frac{2.405}{R}\right)^{2}+\left(\frac{\pi}{L}\right)^{2} .
$$

Так как эксперименты в данной работе проводились в трубке радиусом 2,8 см, а зазор между электродами был равен 2 см, то

$$
\frac{1}{\Lambda^{2}}=\left(\frac{2,405}{R}\right)^{2}+\left(\frac{\pi}{L}\right)^{2}=\left(\frac{2,405}{2,8}\right)^{2}+\left(\frac{\pi}{2}\right)^{2}=4,19 \mathrm{~cm}^{-2} .
$$

Тогда частота диффузионных потерь заряженных частиц равна

$$
v_{d}=\frac{D_{a+}}{\Lambda^{2}}=58,9 \cdot 4,19=246,8 c^{-1},
$$

соответственно, время распада плазмы при давлении 1 Торр равно $\tau_{d}=4,05 \cdot 10^{-3} \mathrm{c}=4,05 \mathrm{mc}$, а при 0,1 Торр оно, соответственно, будет в 10 раз меньше, $\tau_{d}=405 \mu$. Поэтому во всем диапазоне исследованных нами давлений $\mathrm{CO}_{2}$ плазма не успеет заметно распасться из-за амбиполярной диффузии в течение послесвечения.

2. Роль рекомбинации в распадающейся плазме.

Сначала рассмотрим электрон-ионную рекомбинацию, когда происходят потери электронов и положительных ионов $\mathrm{CO}_{2}{ }^{+}$. В работе [33] были исследованы разные виды такой рекомбинации, которые могут описываться следующими реакциями: обычная рекомбинация 
$\begin{array}{ll}\text { и два процесса диссоциативной рекомбинации } & \mathrm{CO}_{2}^{+}+\mathrm{e}^{-} \rightarrow \mathrm{CO}_{2} \\ \mathrm{CO}_{2}^{+}+\mathrm{e}^{-} \rightarrow \mathrm{C}+\mathrm{O}_{2}, \\ \mathrm{CO}_{2}^{+}+\mathrm{e}^{-} \rightarrow \mathrm{CO}+\mathrm{O} .\end{array}$

Авторы [33] выяснили, что реакции (7) и (8) составляют, соответственно, 4\% и 9\% от полного числа актов электрон-ионной рекомбинации, а наиболее вероятной (87\%) является диссоциативная рекомбинация (9). Коэффициент рекомбинации для нее равен $\beta=6,5 \cdot 10^{-7} \mathrm{~cm}^{3} / \mathrm{c}$. Тогда для условий работы [20] частота и время рекомбинационного распада равны $v_{e i}=\beta \cdot n_{+}=6,5 \cdot 10^{-7} \cdot 4,3 \cdot 10^{10}=2,8 \cdot 10^{4} \mathrm{c}^{-1}, \tau_{e i}=36$ мкс. Поэтому в достаточно плотной плазме (как это было в [20]), низкой частоте (20 кГц) и небольших коэффициентах заполнения (когда длительность послесвечения превышает $\tau_{e i}$ ) электрон-ионная рекомбинация может приводить к значительному распаду плазмы.

Однако в плазме в $\mathrm{CO}_{2}$ присутствуют также и отрицательные ионы $\mathrm{O}^{-}$, столкновения которых с положительными ионами $\mathrm{CO}_{2}^{+}$могут приводить к их нейтрализации (ион-ионной рекомбинации):

$$
\mathrm{CO}_{2}^{+}+\mathrm{O}^{-} \rightarrow \mathrm{CO}_{2}+\mathrm{O} \text {. }
$$

К сожалению, в литературе мы не нашли коэффициент такой рекомбинации. Для похожих реакций

$$
\begin{aligned}
& \mathrm{CO}_{2}^{+}+\mathrm{O}_{3}^{-} \rightarrow \mathrm{CO}_{2}+\mathrm{O}_{3}, \\
& \mathrm{O}_{2}^{+}+\mathrm{O}^{-} \rightarrow \mathrm{O}_{2}+\mathrm{O}
\end{aligned}
$$

коэффициенты ион-ионной рекомбинации сильно различаются: $\beta_{i i}=1 \cdot 10^{-6} \mathrm{~cm}^{3} / \mathrm{c}$ для реакции (11) [34] и $\beta_{i i}=2 \cdot 10^{-7} \mathrm{~cm}^{3} / \mathrm{c}$ для реакции (12) [35-37]. Поэтому мы сделаем оценки для двух значений $\beta_{i i}$. Будем предполагать, что для обеих реакций (11) и (12) концентрации положительных ионов равны концентрации ионов $\mathrm{CO}_{2}^{+}$ $\left(4,3 \cdot 10^{10} \mathrm{~cm}^{-3}\right)$, а концентрации отрицательных ионов соответствуют $\mathrm{O}^{-}\left(4 \cdot 10^{10} \mathrm{~cm}^{-3}\right)$ [20]. Тогда при $\beta_{i i}=1 \cdot 10^{-6} \mathrm{~cm}^{3} / \mathrm{c}$ частота ион-ионной рекомбинации $v_{i i}=\beta_{i i} \cdot n_{-}=1 \cdot 10^{-6} \cdot 4 \cdot 10^{10}=4 \cdot 10^{4} \mathrm{c}^{-1}, \tau_{i i}=25$ мкс, а для $\beta_{i i}=2 \cdot 10^{-7} \mathrm{~cm}^{3} / \mathrm{c}$ имеем $v_{i i}=8 \cdot 10^{3} \mathrm{c}^{-1}, \tau_{i i}=125$ мкс. В первом случае ион-ионная рекомбинация может играть существенную роль в распаде плазмы послесвечения, а во втором не должна приводить к заметному уменьшению плотности плазмы. Поэтому этот вопрос остается открытым.

Таким образом, приведенный выше анализ результатов исследований импульсного разряда показывает, что важнейшим фактором в механизме усиления тока в импульсном разряде является нагрев газа в разрядном промежутке в течение плазменной фазы разряда, который приводит к локальному падению концентрации нейтральных молекул и, как следствие, к снижению скорости ионизации. Во избежание терминологической путаницы подчеркнем, что нагрев газа, естественно, ведет к снижению разрядного тока, однако термин "усиление тока", используемый в настоящей работе, относит ток импульсного разряда к току разряда постоянного тока, который всегда меньше вследствие разогрева газа до более высокой температуры.

Еще одним важным вопросом является зависимость разрядного тока от частоты и коэффициента заполнения импульсов. Численное моделирование, проведенное в работе [10], показывает, что в течение нескольких микросекунд после окончания плазменной фазы температура электронов падает с 10-20 эВ в катодном слое и 2-3 эВ в остальной части разряда до очень маленьких величин $0,1-0,2$ эВ. В связи с этим уход заряженных частиц из разряда существенно замедляется, ведь и коэффициенты переноса, и скорость Бома резко падают с уменьшением электронной температуры. Этот вывод подтверждается результатами, показанными на рис. 3 , которые свидетельствуют о том, что при изменении коэффициента заполнения импульсов (следовательно, и интегрального энерговклада в плазму) в 8,5 раз ток разряда меняется всего на 20\%.

Сравнение кривых на рис. 3,4 показывает, что форма осциллограммы тока определяется в первую очередь длительностью плазменной фазы и почти не зависит от длительности фазы послесвечения. Осциллограммы тока при разных частотах и коэффициентах заполнения, но с одинаковой длительностью плазменной фазы, оказываются близки. При этом существует некая критическая длительность импульса (в данном случае около 3 мкс), при превышении которой $I_{p}$ слабо зависит от длительности импульса. Если же длительность импульса ниже критической, то разрядный ток резко падает вплоть до погасания разряда. По-видимому, ключевую роль в формировании плазмы после начала импульса играют первые микросекунды, по истечении которых разряд переходит в квазистационарную фазу.

\section{ВЫВОДЫ}

Целью нашей работы было исследование явления усиления тока в плазменной фазе униполярного импульсного разряда низкого давления в $\mathrm{CO}_{2}$. В настоящей работе экспериментально изучено формирование униполярного импульсного разряда низкого давления (0,1-0,5 Торр) в $\mathrm{CO}_{2}$ в диапазоне частот 20-200 кГц при коэффициенте заполнения импульсов от 0,1 до 0,85 . Основное внимание мы уделяли эффекту усиления тока в импульсном разряде в сравнении с разрядом постоянного тока. Показано, что обнаруженный ранее в среднечастотных микросекундных импульсных разрядах в других газах эффект усиления тока наблюдается также и в разряде в углекислом газе, при этом коэффициент усиления составляет 1-2. Получено, что коэффициент усиления уменьшается с ростом разрядного тока, при увеличении амплитуды импульсного напряжения, а также при повышении давления $\mathrm{CO}_{2}$. Обнаружено, что основное влияние на форму осциллограммы тока импульсного раз- 
ряда оказывает длительность плазменной фазы, в то время как длительность фазы распадающейся плазмы заметной роли не играет. Проведенные оценки показывают, что это обусловлено резким снижением потерь заряженных частиц в связи со значительным падением электронной температуры в фазе послесвечения. Показано, что если длительность импульса мала и ниже некоторой критической величины, то наблюдается резкое уменьшение разрядного тока (вплоть до погасания разряда). Однако при превышении критической длительности импульса (примерно 3 мкс) разрядный ток становится слабо зависящим от длительности импульса. Это дает нам возможность оценить характерное время формирования структуры плазмы после приложения напряжения к электродам.

Анализ процессов, происходящих при формировании униполярного импульсного разряда, показывает, что вклад в процессы, ответственные за формирование разрядного тока, могут вносить диссоциация молекул $\mathrm{CO}_{2}$, образование молекулярного кислорода и отрицательных ионов кислорода. Однако, по нашему мнению, наиболее существенную роль играет нагрев газа в разрядном промежутке (особенно в катодном слое) в течение плазменной фазы разряда, из-за чего локально уменьшаются концентрация молекул газа и скорость их ионизации электронным ударом. В дальнейшем процесс продолжается и приводит к прогреву газа во всем разрядном промежутке и к падению тока разряда до равновесного значения.

Результаты, полученные в настоящей работе, могут быть полезны при разработке эффективных технологий конверсии углекислого газа, которые интенсивно исследуются в настоящее время в связи с необходимостью борьбы с парниковым эффектом в земной атмосфере и перспективами использования в межпланетных космических миссиях.

\section{СПИСОК ЛИТЕРАТУРЫ}

1. Wagenaars E. Plasma breakdown of low-pressure gas discharges // Thesis, Eindhoven: Technische Universiteit Eindhoven, 2006.

2. Efimova V.V., Voronov M.V., Hoffmann V., Eckert J. Electrical Properties of Pulsed Glow Discharge Two New Aspects // Proc. Meeting "Glimmentladungs-Spektroskopie", Berlin, 2008. - P. 27-31.

3. Voronov M., Smid P., Hoffmann V., Hofmann Th., Venzago C. Microsecond pulsed glow discharge in fast flow Grimm type sources for mass spectrometry // J. Anal. At. Spectrom. - 2010. - Vol. 25. - P. 511-518.

4. Voronov M., Hoffmann V., Buscher W., Engelhard C., Rayc S. J., Hieftje G. M. Pressure waves generated in a Grimm-type pulsed glow discharge source and their influence on discharge parameters // J. Anal. At. Spectrom. - 2011. - Vol. 26. P. 811-815.

5. Efimova V., Hoffmann V., Eckert J. Electrical properties of the ms pulsed glow discharge in a Grimm-type source: comparison of DC and RF modes // J. Anal. At. Spectrom. - 2011. - Vol. 26. - P. 784-791.

6. Voronov M., Hoffmann V., Buscher W., Engelhard C., Rayc S.J., Hieftje G.M. Thermal mechanism for formation of electrical prepeak and pressure waves in a microsecond direct current pulsed glow discharge with a Grimm-type source: a modeling investigation // J. Anal. At. Spectrom. - 2012. - Vol. 27. - P. 1225-1233.

7. Gamez G., Bogaerts A., Hieftje G. M. Temporal and spatially resolved laser-scattering plasma diagnostics for the characterization of a ms-pulsed glow discharge // J. Anal. At. Spectrom. - 2006. - Vol. 21. - P. 350-359.

8. Lisovskiy V.A., Ogloblina P.A., Dudin S.V., Yegorenkov V.D., Dakhov A.N. Forming a unipolar pulsed discharge in nitrogen // Problems of atomic science and technology. - 2016. - No.6. - P. 227-230.

9. Lisovskiy V.A., Ogloblina P.A., Dudin S.V., Yegorenkov V.D., Dakhov A.N., Farenik V.I. Formation stages of pulsed discharge in oxygen and carbon tetrafluoride // Journal of Surface Physics and Engineering. - 2016. - Vol. 1. - No. 4. P. 351-360.

10. Lisovskiy V.A., Ogloblina P.A., Dudin S.V., Yegorenkov V.D., Dakhov A.N. Current gain of a pulsed de discharge in lowpressure gases // Vacuum. - 2017. - Vol.145. - P. 194-202.

11. Berenguer Ch., Katsonis K. Global Modeling of $\mathrm{CO}_{2}$ Discharges with Aerospace Applications // Advances in Aerospace Engineering. - 2014. - Article ID 847097. - 17p.

12. Kozak T., Bogaerts A. Splitting of $\mathrm{CO}_{2}$ by vibrational excitation in non-equilibrium plasmas: a reaction kinetics model // Plasma Sources Sci. Technol. - 2014. - Vol.23. - No.4. - P. 045004.

13. De Bie Ch., van Dijk J., Bogaerts A. The Dominant Pathways for the Conversion of Methane into Oxygenates and Syngas in an Atmospheric Pressure Dielectric Barrier Discharge // J. Phys. Chem. C. - 2015. - Vol. 119. - P. 22331-22350.

14. Bogaerts A., Kozak T., van Laer K., Snoeckx R. Plasma-based conversion of $\mathrm{CO}_{2}$ : current status and future challenges // Faraday Discuss. - 2015. - Vol. 183. - P. 217-232.

15. Van Rooij G.J., van den Bekerom D.C.M., den Harder N., Minea T., Berden G., Bongers W.A., Engeln R., Graswinckel M.F., Zoethout E., van de Sanden M.C.M. Taming microwave plasma to beat thermodynamics in $\mathrm{CO}_{2}$ dissociation // Faraday Discuss. -2015 . -16 p. (DOI: 10.1039/c5fd00045a).

16. Aerts R., Somers W., Bogaerts A. Carbon Dioxide Splitting in a Dielectric Barrier Discharge Plasma: A Combined Experimental and Computational Study // ChemSusChem. - 2015. - Vol. 8. - P. $702-716$.

17. Snoeckx R., Heijkers S., Van Wesenbeeck K., Lenaerts S., Bogaerts A. $\mathrm{CO}_{2}$ conversion in a dielectric barrier discharge plasma: $\mathrm{N}_{2}$ in the mix as a helping hand or problematic impurity? // Energy Environ. Sci. - 2016. - Vol.9. - P. 999-1011.

18. Guerra V., Silva T., Ogloblina P., Grofulović M., Terraz L., da Silva M.L., Pintassilgo C.D., Alves L.L., Guaitella O. The case for in situ resource utilisation for oxygen production on Mars by nonequilibrium plasmas // Plasma Sources Sci. Technol. 2017. - Vol.26. - P. 11LT01.

19. Diver D.A. A plasma formulary for physics, technology and astrophysics. - Berlin: Wiley, 2001. $-207 \mathrm{p}$.

20. Berenguer Ch., Katsonis K. Global Modeling of CO2 Discharges with Aerospace Applications // Advances in Aerospace Engineering. - 2014. - P. 847097. 
21. Massey H. Negative ions. - London: Cambridge University Press, 1976. - 754 p.

22. Schulz G.J. Cross sections and electron affinity for $\mathrm{O}^{-}$ions from $\mathrm{O}_{2}, \mathrm{CO}$, and $\mathrm{CO}_{2}$ by electron impact // Phys. Rev. - 1962. Vol. 128. - No. 1. - P. 178-186.

23. Raizer Yu.P. Gas discharge physics. - Berlin: Springer, 1991. - 449 p.

24. Martin A., Bordel N., Pereiro R., Bogaerts A. Monte Carlo analysis of the electron thermalization process in the afterglow of a microsecond dc pulsed glow discharge // Spectrochimica Acta, Part B. - 2008. - Vol.63. - P.1274-1282.

25. Lisovskiy V., Yegorenkov V. Ambipolar diffusion in strongly electronegative plasma // Europhysics Letters. - 2012. - Vol.99. - No.3. - P. $35002(1-5)$

26. Thompson J.B. Negative Ions in the Positive Column of the Oxygen Discharge // Proc. Phys. Soc. - 1959. - Vol.73, No.5. P. 818-821.

27. Lichtenberg A.J., Vahedi V., Lieberman M.A., Rognlien T. Modeling electronegative plasma discharges // J. Appl. Phys. 1994. - Vol.75, No.5. - p. 2339-2347.

28. Grofulović M., Alves L.L., Guerra V. Electron-neutral scattering cross sections for CO2: a complete and consistent set and an assessment of dissociation // J. Phys. D: Appl. Phys. - 2016. - Vol. 49. - P. 395207.

29. Schultz G., Charpak G., Sauli F. Mobilities of positive ions in some gas mixtures used in proportional and drift chambers // Revue de Physique Appliquee. - 1977. - Vol. 12. - No. 1. - P. 67-70.

30. Saporoschenko M. Drift Velocities of $\mathrm{O}^{+}, \mathrm{CO}^{+}$, and $\mathrm{CO}_{2}^{+}$Ions in $\mathrm{CO}_{2}$ Gas // Phys. Rev. A. - 1973. - Vol.8. - No.2. P. 1044-1047.

31. McKnight L.G. Drift velocities and interactions of negative ions in oxygen // Phys. Rev. A. - 1970. - Vol. 2. - No. 3. P. 762-770.

32. Snuggs R.M., Volz D.J., Schummers J.H., Martin D.W., McDaniel E.W. Mobilities and longitudinal diffusion coefficients of mass-identified potassium ions and positive and negative oxygen ions in oxygen // Phys. Rev. A. - 1971. - Vol. 3. - No. 1. P. 477-487.

33. Seiersen K., Al-Khalili A., Heber O., Jensen M.J., Nielsen I.B., Pedersen H.B., Safvan C.P., Andersen L.H. Dissociative recombination of the cation and dication of $\mathrm{CO}_{2} / /$ Phys. Rev.A. - 2003. - Vol. 68. - No.2. - P. 022708.

34. Mesyats G.A., Osipov V.V., Tarasenko V.F. Pulsed Gas Lasers. - Bellingham, WA: Opt. Eng. Press, 1995. - 272 p.

35. Bekefi J. Principles of laser plasma. - New York: Wiley, 1976. - 416p.

36. Moseley J.T., Abertha W., Peterson J.R. Two-body mutual neutralization rates of $\mathrm{O}_{2}^{+}+\mathrm{O}^{-}, \mathrm{NO}^{+}+\mathrm{O}^{-}$, and $\mathrm{Na}^{+}+\mathrm{O}^{-}$obtained with merged beams // J. Geophysical Research. - 1972. - Vol.77. - No.1. - P. 255-259.

37. Smith K., Thomson R.M. Computer modeling of gas lasers. - New York: Plenum Press, 1978. - 515 p. 